\title{
İki Boyutlu Bazı Datum Dönüşüm Yöntemlerinin İncelenmesi
}

\author{
Mustafa HÜSREVOĞLU ${ }^{1 *}$, Ekrem TUŞAT ${ }^{2}$ \\ ${ }^{1}$ Selçuk Üniversitesi, Fen Bilimleri Enstitüsü, Harita Mühendisliği Bölümü, Konya \\ (mustafahusrev@gmail.com) ORCID ID 0000 - 0003 - 1324 - 9617 \\ ${ }^{2}$ Selçuk Üniversitesi, Mühendislik Fakültesi, Harita Mühendisliği Bölümü, Konya \\ (etusat@selcuk.edu.tr) ORCID ID 0000 -0003-4130-3764
}

\begin{abstract}
Öz
Birçok mühendislik projesi, kuruluş ve ülkeler yeryüzündeki konumları nedeniyle veya çalışma amaçlarına göre birbirlerinden farklı koordinat sistemleri kullanır. Günümüzde genellikle, daha önce yerel koordinat sistemleri ile belirlenmiş olan konum değerlerinin ve harita altlıklarının, global koordinat sistemleri üzerindeki karşılıklarına ihtiyaç duyulmaktadır. Bu durum datum dönüşümü yapmayı gerektirmektedir. Çeşitli koordinat sistemlerine ait verilerin diğer bir sistemdeki karşıllı̆ı bulunmak istendiğinde datum dönüşümü uygulanır. Datum dönüşümleri yapılırken benzerlik, afin ve projektif dönüşüm yöntemleri kullanılabilir. Her bir yöntem için ayrı işlem adımları uygulanır ve aynı işlem için sonuçlar farklı olabilir. Arazi koordinatlarının dönüşümünde benzerlik dönüşümü yaygın olarak kullanılırken, fotogrametri ile kartografya alanlarında afin ve projektif dönüşüm yöntemleri sıklıkla kullanılır. Bu çalışmada iki farklı koordinat sistemindeki karşılıkları bilinen noktalar ile arazi koordinatlarının iki boyutlu dönüşüm uygulamaları incelenmiştir. Hesaplamalar MS Excel ortamında yapılmış olup dayanak noktalarının konumları ile uygulanan yöntemler arasında karşılaştırmalar yapılmıştır. Uygulamalar sonucunda arazi koordinatlarının dönüşümünde projektif dönüşüm yönteminin uygun olabileceği belirlenmiştir.
\end{abstract}

Anahtar Kelimeler: datum dönüşümleri, koordinat dönüşümleri, benzerlik dönüsümü, afin dönüşümü, projektif dönüşüm

\section{Examination of Some Two-Dimensional Datum Transformations Methods}

\begin{abstract}
Many engineering projects, organizations and countries use different coordinate systems because of their position on the earth or for their working purposes. Today, there is a need for correspondences, on global coordinate systems, of the position values and map bases previously determined using local coordinate systems. This requires datum transformation. Datum transformation is applied when data of various coordinate systems are to be provided in another system. Similarity, affine and projective transformation methods can be used when datum transformations are made. Separate process steps are applied for each method and the results may be different for the same process. While similarity transformation is widely used in the transformation of land coordinates, affine and projective transformation methods are frequently used in the fields of photogrammetry and cartography. In this study, the applications of two-dimensional transformations of the known points and the terrain coordinates in two different coordinate systems were examined. Calculations were made in MS Excel environment and the positions of the reference points were compared with the applied methods. As a result of the applications, it has been determined that the projective transformation method may be suitable for transforming the terrain coordinates.
\end{abstract}

Keywords: datum transformations, coordinate transformations, similarity transformation, affine transformation, projective transformation

* Sorumlu Yazar 


\section{GİRİş}

Dünya çapında birçok ülke tarafından haritacılık ve ölçüm işleri için, yüzlerce yerel jeodezik koordinat sistemi geliştirilmiştir (Mitsakaki, Agatza-Balodimou, \& Papazissi, 2006; Vaníček \& Steeves, 1996). WGS84 (World Geodetic System 1984) gibi global jeosentrik datumlar kullanan uydularla konum belirleme (GNSS) sistemlerinin hızlı gelişimi, yaygın kullanımı ve ölçüm işlerinde rutine dönüşmesi, lokal koordinat sistemleri kullanan ülkelerin global referans sistemleri ile kendi datum sistemleri arasında dönüşüm yapma ihtiyacını ve ilgisini artırdı. Uydu teknolojileri ile birlikte kullanılan global jeosentrik datumlar, jeodezik kontrol ve gözlem ağlarının yaygın datumu olmaya başlamıştır. Ülkeler uydularla konum belirleme sistemlerinden faydalanmak için mevcut verileri ile sürekli olarak dönüşüm yapmakta veya tamamen datumlarını değiştirmektedirler. Uydu teknolojilerinin gelişimi ve eski haritaların güncellenmesi ihtiyaci ile birlikte datum dönüşümlerinin kullanımı ve önemi artmıştır (Even-Tzur, 2000; Hofmann-Wellenhof, Lichtenegger, \& Wasle, 2007; Kwon, Bae, Choi, Lee, \& Lee, 2005; Mitsakaki ve diğ., 2006; Ogaja, 2011).

Datum ve datum dönüşümü terimlerin sözlük karşıl1kları şöyle verilmiştir;

Datum: 'Diğer niceliklerin hesaplanabilmesi için referans veya temel oluşturan bir grup ya da herhangi bir niceliktir. Kullanılan bir koordinat sistemini belirlemek-tanımlamak için gereken tüm nicelikleri içeren bir referanstır. Jeodezik kontrol için kullanilan koordinat sistemini belirten bir sabitler setidir'(Geodetic Glossary, 1986).

Datum dönüşümü: 'Farklı datumların nirengi ağları arasındaki bitişiklik ve örtüşme farklıliklarının sistematik olarak elimine edilmesi ve ă̆ların döndürülerek, ölçeklenerek, merkezlerinin taşınarak birbirleri ile uygun hale getirilmesi işlemine datum dönüşümü denir'(A DOD Glossary of Mapping, Charting and Geodetic Terms, 1967).

Yeryüzü üzerinde konumların belirlenebilmesi için çeşitli koordinat sistemleri tanımlanmıştır.
Farkl1 koordinat sistemlerine ait koordinat verilerinin ortak bir koordinat sistemindeki karşılığının belirlenmesi istendiğinde datum dönüşümü uygulanır. Datum kelimesi, "hesaplamalarda temel olarak kullanılması kabul edilen yüzeyler için temel bilgi veya başlangıç yüzeyi ile ilgili sabit bilgiler" olarak tanımlanabilir (Şişman \& Dilaver, 2005). Boyutları belirlenmiş bir elipsoit ve jeoit arasındaki ilişkinin kurulmasına 'Jeodezik Datum' adı verilir (Üstün, 1996). Datum ve referans koordinat sistemi tanımlamak aynıdır (Uzun, 2003). Bir jeodezik ağın datumu ile hem ağın temelini oluşturan referans elipsoidi hem de jeodezik ağ noktalarının koordinatının elde edileceği koordinat sistemi tanımlanmış olur. Bu nedenle iki datum arasındaki datum dönüşümü ile iki koordinat sistemi arasındaki koordinat dönüşümü aynı işlem olur (Aksoy, 1999). Koordinat dönüşümünde iki koordinat sistemi arasındaki dönüşüm parametreleri her iki sistemde de koordinatları bilinen ortak noktalardan hesaplanır. Gereğinden fazla ortak nokta bulunması durumunda bu işlem 'En Küçük Kareler' (EKK) yöntemi ile gerçekleştirilir (Akyılmaz, Acar, \& Özlüdemir, 2007). Datum dönüşümleri iki yada üç boyutlu olarak yapılabilir. Dönüşüm doğruluğu genel olarak ortak noktaların sayısı ve dağılımına, her iki sistemdeki konum doğruluklarına ve kullanılan dönüşüm modeline bağlıdır (Deniz ve diğ., 2012). Bu çalışmada iki boyutlu benzerlik, afin ve projektif dönüşüm yöntemleri uygulamalı olarak incelenmiştir. Uygulama için gerekli hesaplamalar MS Excel yazılımı aracılığı ile yapılmıştır. Farklı yöntemlere ait sonuçlar ve dayanak noktası olarak belirlenen noktaların konumları dikkate alınarak sonuç veriler incelenmiştir.

\section{1. İki Boyutlu Datum Dönüşümleri}

İki elipsoidal datum arasındaki farklar; koordinat başlangıç nokta konumlarının farklı olmas1, koordinat eksenlerinin yönlendirmesinin farklı olması ve referans elipsoidinin büyüklüğü ve şeklinin farklı olmas1 nedenleriyle meydana gelmektedir (Kahveci \& Y1ld1z, 2012). Teorik olarak iki koordinat sistemi arsındaki dönüşüm, en basit şekilde kartezyen koordinatlar ile ifade edilir, eğer konumlar eğri koordinatlar ile ifade edilmiş ise denklemlerin kullanılabilmesi için ve dönüşüm hesaplamalarında netlik olması adına ilk önce koordinatlar kendi sistemlerinde 
temsil ettikleri kartezyen koordinat formuna dönüştürülür (Featherstone \& Vanicek, 1999; Vaníček \& Steeves, 1996). Bu işleme koordinat dönüştürme denilmektedir. Datum dönüşümü kartezyen koordinatlar ile uygulanırken iki sistem arasındaki geometrik ilişki ortaya çıkar ve böylece dönüşüm yapilırken herhangi bir referans sistemine ait özellikleri dikkate almak gerekmez.

Dönüşüm işlemi benzerlik, afin ve projektif yöntemleri ile yapılabilir. Her yöntem farklı sonuçlar verebilir ve parametre değerleri farklıdır. Uygulanacak yöntem, yapılacak çalışmalara uygun şekilde seçilir.

\subsubsection{Benzerlik dönüşümü}

İki boyutlu benzerlik dönüşümü, Helmert dönüşümü olarak ta bilinir. Benzerlik dönüşümünden sonra gerçek şekil korunur ve genellikle ayrı referans sistemlerinde yapılan ölçümlerin ortak bir referans sistemine dönüşümü için kullanılır (Ghilani, 2011). Benzerlik dönüşümü işlemini Ghilani üç ayrı adımla açıklamıştır, bu adımlar; iki sistemde eşit boyutlar oluşturmak için ölçekleme, iki sistemin eksenlerinin paralel olması için döndürme ve ortak bir orijin oluşturmak için yapılacak ötelemedir. Ölçekleme ve döndürme için ölçek faktörü $(\mathrm{m})$, dönüklük açısı $(\varepsilon)$ olmak üzere iki parametre belirlenmiştir. Bu parametrelere ek olarak yukarı ve sağa yönde yapılacak öteleme işlemi için ise iki ayrı parametre belirlenir. Böylece benzerlik dönüşümü için bu dört parametrenin hesaplanmas1 gerekir. Dört parametrenin çözümü için bu dört parametreye karş1lık gelen her iki sistemde koordinatları bilinen en az iki ortak noktaya ihtiyaç vardır (Başçiftçi \& İnal, 2008).

Birinci sistem koordinatları : $\mathrm{x}$, y ve ikinci sistem koordinatları $: \mathrm{X}, \mathrm{Y}$ olmak üzere iki koordinat sistemi arasında uygulanacak benzerlik dönüşümünde kullanılacak bağıntı, denklem ve dönüşüm matrisleri (1-9) (Ghilani, 2011);

$\mathrm{X}=\mathrm{ax}-\mathrm{by}+\mathrm{c}$

$\mathrm{Y}=\mathrm{ay}+\mathrm{bx}+\mathrm{d}$

olmak üzere ;

$a=m \cdot \cos \varepsilon, b=m . \sin \varepsilon$, m:ölçek faktörü, $\varepsilon$ :iki sistem arasındaki dönüklük açısı. $m=\sqrt{a^{2}+b^{2}}$

$\tan \varepsilon=\frac{a}{b}$

Koordinatların düzeltme denklemleri;

$$
\begin{aligned}
& \mathrm{ax}_{1}-\mathrm{by}_{1}+\mathrm{c}=\mathrm{X}_{1}+\mathrm{V}_{\mathrm{X} 1} \\
& \mathrm{ay}_{1}+\mathrm{bx}_{1}+\mathrm{d}=\mathrm{Y}_{1}+\mathrm{V}_{\mathrm{Y} 1} \\
& \mathrm{ax}_{2}-\mathrm{by}_{2}+\mathrm{c}=\mathrm{X}_{2}+\mathrm{V}_{\mathrm{X} 2} \\
& \mathrm{ay}_{2}+\mathrm{bx}_{2}+\mathrm{d}=\mathrm{Y}_{2}+\mathrm{V}_{\mathrm{Y} 2} \\
& \cdots \\
& \mathrm{ax}_{\mathrm{n}}-\mathrm{by}_{\mathrm{n}}+\mathrm{c}=\mathrm{X}_{\mathrm{n}}+\mathrm{V}_{\mathrm{Xn}} \\
& \mathrm{ay}_{\mathrm{n}}+\mathrm{bx}_{\mathrm{n}}+\mathrm{d}=\mathrm{Y}_{\mathrm{n}}+\mathrm{V}_{\mathrm{Yn}}
\end{aligned}
$$

Parametrelerin hesabı için kullanılacak dönüşüm matrisleri;

$\mathrm{A}=\left[\begin{array}{llll}x_{1} & -y_{1} & 1 & 0 \\ y_{1} & x_{1} & 0 & 1 \\ \cdots & \cdots & \cdots & \cdots \\ \cdots & \cdots & \cdots & \cdots \\ x_{n} & -y_{n} & 1 & 0 \\ y_{n} & x_{n} & 0 & 1\end{array}\right]_{2 n X 4}$

$\mathrm{X}=\left[\begin{array}{c}a \\ b \\ c \\ d\end{array}\right]_{4 X 1} \mathrm{~L}=\left[\begin{array}{c}X_{1} \\ Y_{1} \\ \cdots \\ \cdots \\ X_{n} \\ Y_{n}\end{array}\right]_{2 n X 1} \quad \mathrm{~V}=\left[\begin{array}{c}V_{X_{1}} \\ V_{Y_{1}} \\ \cdots \\ \cdots \\ V_{X_{n}} \\ V_{Y_{n}}\end{array}\right]_{2 n X 1}$

$\mathrm{V}=\mathrm{AX}-\mathrm{L}$

a,b,c ve $d$ bilinmeyenlerinin bulunması için $X$ matrisi hesaplanır;

$\mathrm{N}=\mathrm{A}^{\mathrm{T}} \mathrm{PA}, \mathrm{n}=\mathrm{A}^{\mathrm{T}} \mathrm{PL}, \mathrm{X}=\mathrm{N}^{-1} \mathrm{n}$

eşitliği ile bulunur. Koordinatların ortalama hatasi;

$m_{o}=m_{X}=m_{Y}= \pm \sqrt{\frac{V_{X}^{2}+V_{Y}^{2}}{2 n-4}}$

bir P noktasının konum hatas1; 
$m_{p}=m_{o} \sqrt{2}= \pm \sqrt{\frac{V_{X}^{2}+V_{Y}^{2}}{n-2}}$

xy sisteminde bir kare benzerlik dönüşümünden sonra kare olarak kalır, ancak ötelenmiş, dönmüş ve ölçek olarak değişmiştir. Buna karşın bu karenin afin dönüşümünden sonra bir paralel kenar elde edilir (Kraus, 2007).

\subsubsection{Afin dönüşümü}

Afin dönüşüm hesaplamalarında altı parametre kullanılır. Bunlar, yukarı ve sağa yönde ayrı ayrı olarak belirlenen iki ölçek faktörü, iki dönüklük ve iki öteleme parametreleridir. Parametrelerin çözümü için en az üç ortak nokta gerekir ve üçten fazla nokta mevcut ise en küçük kareler yöntemi uygulanabilir. Afin dönüşüm genellikle basılı haritaların sayısallaştırılması, fotogrametri uygulamalarında ve resim koordinat sistemlerinin dönüşümünde kullanılır. Bunun sebebi yukarı ve sağa yönde iki ayrı ölçek ve dönüklük parametresinin belirlenmesidir.

Birinci sistem koordinatları : $\mathrm{x}, \mathrm{y}$ ve ikinci sistem koordinatları : $\mathrm{X}, \mathrm{Y}$ olmak üzere iki koordinat sistemi arasinda uygulanacak afin dönüşümünde kullanılacak bağınt1, denklem ve dönüşüm matrisleri (10-16) (Ghilani, 2011);

$X=a x+b y+c$

$\mathrm{Y}=\mathrm{dx}+\mathrm{ey}+\mathrm{f}$

olmak üzere, koordinatların düzeltme denklemleri;

$\mathrm{ax}_{1}+\mathrm{by}_{1}+\mathrm{c}=\mathrm{X}_{1}+\mathrm{V}_{\mathrm{X} 1}$

$\mathrm{dx}_{1}+\mathrm{ey}_{1}+\mathrm{f}=\mathrm{Y}_{1}+\mathrm{V}_{\mathrm{Y} 1}$

$\mathrm{ax}_{2}+\mathrm{by}_{2}+\mathrm{c}=\mathrm{X}_{2}+\mathrm{V}_{\mathrm{X} 2}$

$\mathrm{dx}_{2}+\mathrm{ey}_{2}+\mathrm{f}=\mathrm{Y}_{2}+\mathrm{V}_{\mathrm{Y} 2}$

$a_{n}+b y_{n}+c=X_{n}+V_{X n}$

$\mathrm{dx}_{\mathrm{n}}+\mathrm{ey}_{\mathrm{n}}+\mathrm{f}=\mathrm{Y}_{\mathrm{n}}+\mathrm{V}_{\mathrm{Yn}}$

Parametrelerin hesabı için kullanılacak dönüşüm matrisleri;

$$
\mathrm{A}=\left[\begin{array}{cccccc}
x_{1} & y_{1} & 1 & 0 & 0 & 0 \\
0 & 0 & 0 & x_{1} & y_{1} & 1 \\
\cdots & \cdots & \cdots & \cdots & \cdots & \cdots \\
\cdots & \cdots & \cdots & \cdots & \cdots & \cdots \\
x_{n} & y_{n} & 1 & 0 & 0 & 0 \\
0 & 0 & 0 & x_{n} & y_{n} & 1
\end{array}\right]_{2 n X 6}
$$

$\mathrm{X}=\left[\begin{array}{c}a \\ b \\ c \\ d \\ e \\ f\end{array}\right]_{6 X 1} \quad \mathrm{~L}=\left[\begin{array}{c}X_{1} \\ Y_{1} \\ \cdots \\ \cdots \\ X_{n} \\ Y_{n}\end{array}\right]_{2 n X 1} \quad \mathrm{~V}=\left[\begin{array}{c}V_{X_{1}} \\ V_{Y_{1}} \\ \cdots \\ \cdots \\ V_{X_{n}} \\ V_{Y_{n}}\end{array}\right]_{2 n X 1}$

$\mathrm{V}=\mathrm{AX}-\mathrm{L}$

$\mathrm{a}, \mathrm{b}, \mathrm{c}, \mathrm{d}, \mathrm{e}, \mathrm{f}$ bilinmeyenlerinin bulunması için $\mathrm{X}$ matrisi hesaplanır;

$\mathrm{N}=\mathrm{A}^{\mathrm{T}} \mathrm{PA}, \mathrm{n}=\mathrm{A}^{\mathrm{T}} \mathrm{PL}, \mathrm{X}=\mathrm{N}^{-1} \mathrm{n}$

Koordinatların ortalama hatas1;

$m_{o}=m_{X}=m_{Y}= \pm \sqrt{\frac{V_{X}^{2}+V_{Y}^{2}}{2 n-6}}$

bir P noktasının konum hatasi;

$m_{p}=m_{o} \sqrt{2}= \pm \sqrt{\frac{V_{X}^{2}+V_{Y}^{2}}{n-3}}$

\subsubsection{Projektif dönüşüm}

Projektif dönüşümde sekiz parametrenin çözümü için her iki sistemde koordinatları bilinen en az dört eşlenik noktaya ihtiyaç duyulmaktadır (Başçiftçi \& İnal, 2008). Bu dönüşüm yöntemi fotogrametri alanında yaygın olarak kullanılmakla birlikte afin dönüşüm ile benzerlik gösterir.

Projektif dönüşüm uygulanırken $a_{1}, b_{1}, c_{1}, a_{2}$, $\mathrm{b}_{2}, \mathrm{c}_{2}, \mathrm{a}_{3}, \mathrm{~b}_{3}$ parametrelerinin hesab1 için ilk olarak afin dönüşüm yapılır, afin dönüşüm ile $a_{1}, b_{1}, \quad c_{1}, a_{2}, b_{2}, c_{2}$ parametrelerinin ilk değerleri hesaplanmış olur. Projektif dönüşüme devam ederken $a_{3}=b_{3}=0$ olarak kabul edilir ve dönüşüm matrislerine uygulanır, $a_{3}$ ve $b_{3}$ parametreleri bulunduktan sonra iteratif olarak sekiz parametre arasında fark görülmeyinceye 
kadar işlem sürdürülür. Parametrelerin yeni değerleri ile ikinci sistemin koordinatları belirlenir.

Projektif dönüşümde A matrisi oluşturulurken $\mathrm{x}$ ve $\mathrm{y}$ değerlerinden birinci sistem ortak noktaların ortalaması $\mathrm{x}_{0}$ ve $\mathrm{y}_{0}$ değerlerinin farkı alınarak işleme devam edilir aynı şekilde $\mathrm{L}$ matrisi ise $\mathrm{X}$ ve $\mathrm{Y}$ değerleri ile ikinci sistem ortak noktalarının ortalaması $X_{o}$ ve $Y_{o}$ farkları ile oluşturulur. $\mathrm{Bu}$ bakımdan projektif dönüşüme başlamadan önce yapılacak afin dönüşümde de $\mathrm{A}$ ve $\mathrm{L}$ matrisleri oluşturulurken bu işlemler uygulanmalıdır.

Projektif dönüşüm için kullanılacak bağıntı, denklem ve dönüşüm matrisleri (17-23) (Ghilani, 2011);

$$
\begin{aligned}
& X=\frac{a_{1} x+b_{1} y+c_{1}}{a_{3} x+b_{3} y+1}+X_{o} \\
& Y=\frac{a_{2} x+b_{2} y+c_{2}}{a_{3} x+b_{3} y+1}+Y_{o}
\end{aligned}
$$

Parametrelerin hesabı için kullanılacak dönüşüm matrisleri;

A matrisi;

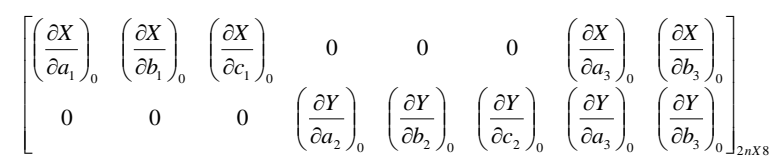

$$
\frac{\partial X}{\partial a_{1}}=\frac{x}{a_{3} x+b_{3} y+1} \quad \frac{\partial Y}{\partial a_{2}}=\frac{x}{a_{3} x+b_{3} y+1}
$$$$
\frac{\partial X}{\partial b_{1}}=\frac{y}{a_{3} x+b_{3} y+1} \quad \frac{\partial Y}{\partial b_{2}}=\frac{y}{a_{3} x+b_{3} y+1}
$$$$
\frac{\partial X}{\partial c_{1}}=\frac{1}{a_{3} x+b_{3} y+1} \quad \frac{\partial Y}{\partial c_{2}}=\frac{1}{a_{3} x+b_{3} y+1}
$$$$
\frac{\partial X}{\partial a_{3}}=-\frac{a_{1} x+b_{1} y+c_{1}}{\left(a_{3} x+b_{3} y+1\right)^{2}} x
$$

$$
\begin{aligned}
& \frac{\partial X}{\partial b_{3}}=-\frac{a_{1} x+b_{1} y+c_{1}}{\left(a_{3} x+b_{3} y+1\right)^{2}} y \\
& \frac{\partial Y}{\partial a_{3}}=-\frac{a_{2} x+b_{2} y+c_{2}}{\left(a_{3} x+b_{3} y+1\right)^{2}} x \\
& \frac{\partial Y}{\partial b_{3}}=-\frac{a_{2} x+b_{2} y+c_{2}}{\left(a_{3} x+b_{3} y+1\right)^{2}} y
\end{aligned}
$$

$\mathrm{X}=\left[\begin{array}{c}a_{1_{0}} \\ b_{1_{0}} \\ c_{1_{0}} \\ a_{2_{0}} \\ b_{2_{0}} \\ c_{2_{0}} \\ a_{3_{0}} \\ b_{3_{0}}\end{array}\right]_{8 X 1} \quad\left[\begin{array}{c}X_{1}-X_{o} \\ Y_{1}-Y_{o} \\ \cdots \\ \cdots \\ X_{n}-X_{o} \\ Y_{n}-Y_{o}\end{array}\right]_{2 n X 1}$

$\mathrm{V}=\mathrm{AX}-\mathrm{L}$

$a_{1}, b_{1}, c_{1}, a_{2}, b_{2}, c_{2}$ parametrelerinin afin dönüşümü ile hesaplanmasının ardından, $\mathrm{a}_{3}=$ $\mathrm{b}_{3}=0$ kabul edilerek projektif dönüşüm için ilk A matrisi oluşturulur ve $X$ matrisi hesaplanır. İşlemler sonucunda hesaplanan X matrisi ile yeni parametre değerleri elde edilir ve yeniden A matrisi oluşturularak, $\mathrm{X}$ matrisi hesaplanır. $\mathrm{Bu}$ işlem her işlem sonucunda elde edilen parametreler ile önceki işleme ait parametreler arasında fark görülmeyinceye kadar iteratif olarak sürdürülür. Elde edilen parametreler ile ikinci sisteme ait koordinatlar hesaplanır.

Koordinatların ortalama hatas1;

$m_{o}=m_{X}=m_{Y}= \pm \sqrt{\frac{V_{X}^{2}+V_{Y}^{2}}{2 n-8}}$

bir P noktasının konum hatası;

$m_{p}=m_{o} \sqrt{2}= \pm \sqrt{\frac{V_{X}^{2}+V_{Y}^{2}}{n-4}}$ 


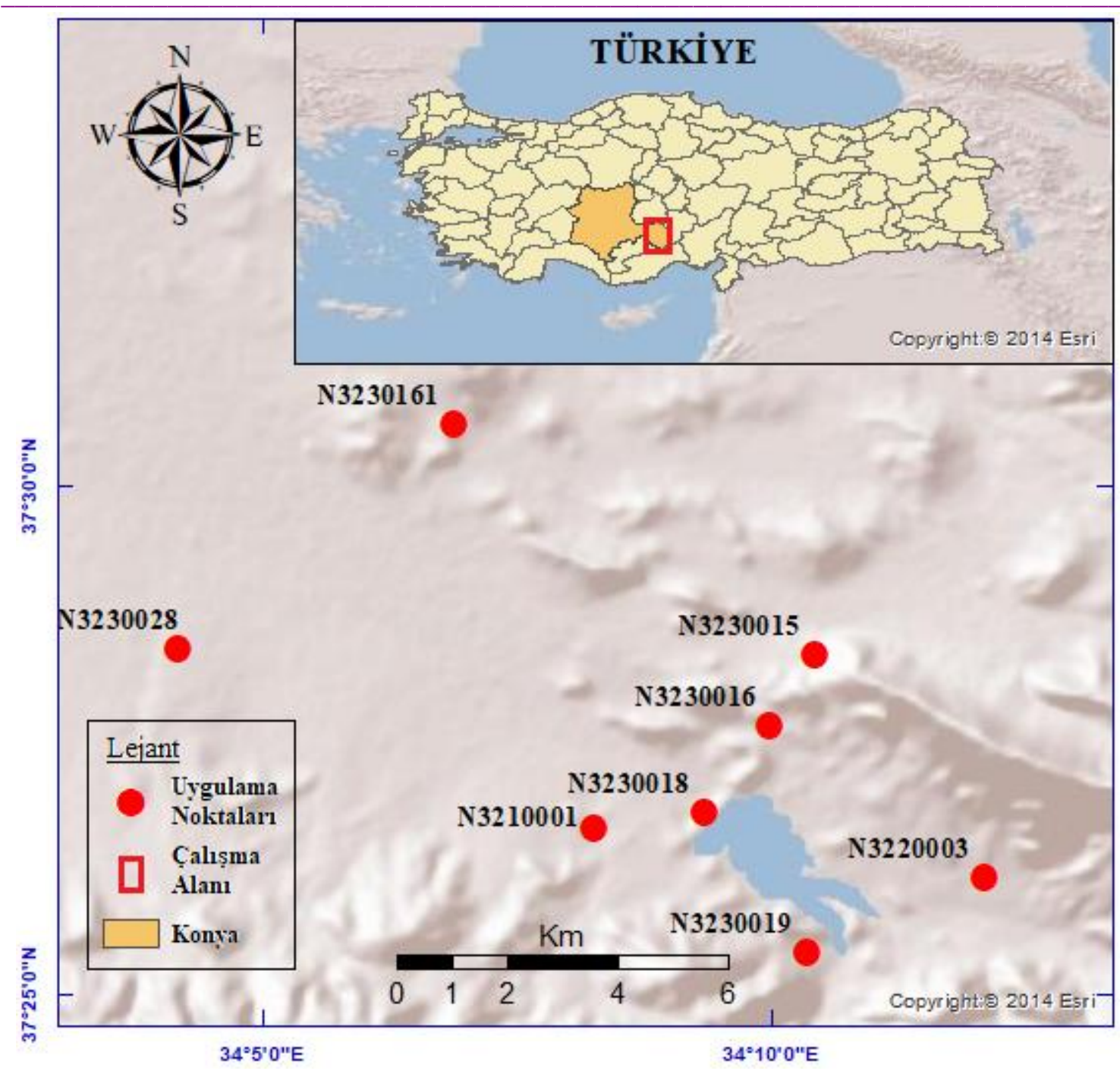

Şekil 1. Uygulama Noktalarının Konumları

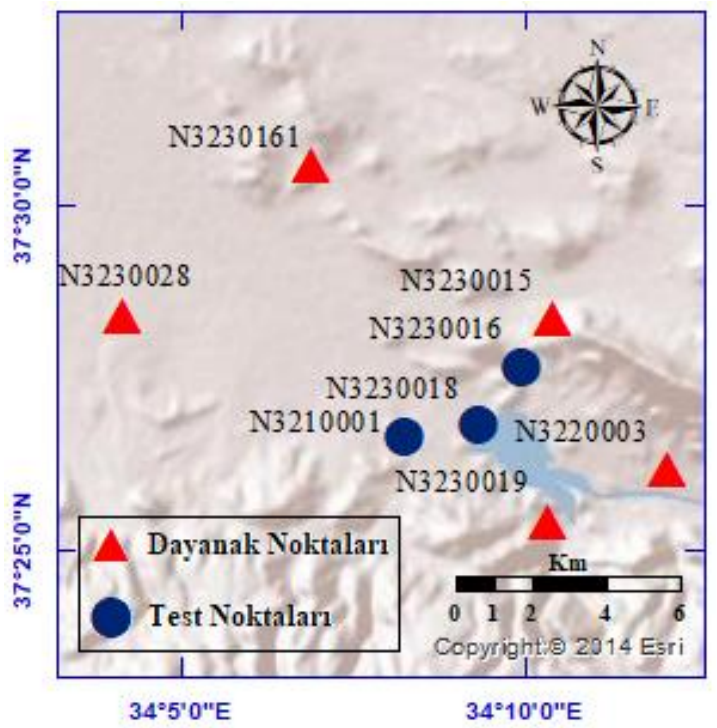

Şekil 2. Uygulama-1 için Dayanak ve Test Noktalarının Konumları

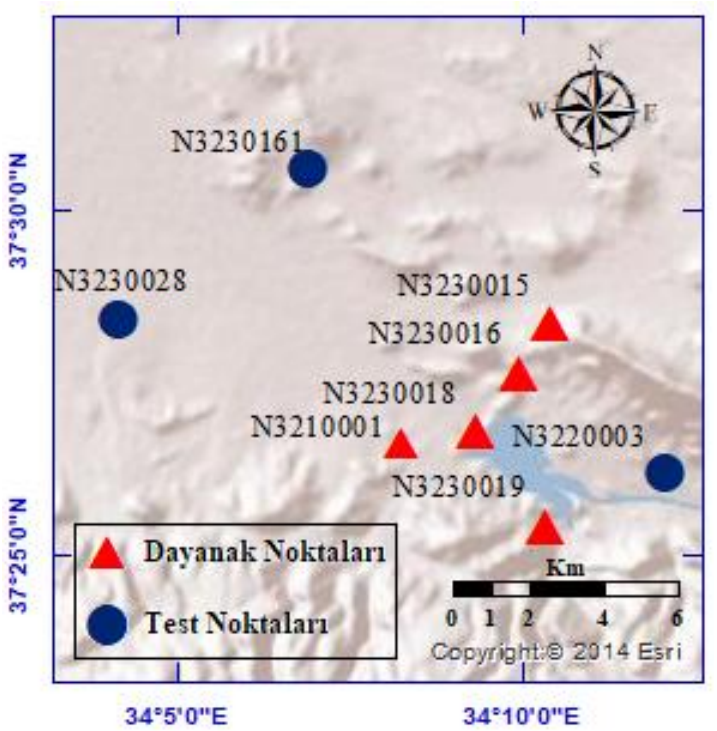

Şekil 3. Uygulama-2 için Dayanak ve Test Noktalarının Konumları 


\section{UYGULAMA}

$\mathrm{Bu}$ bölümde benzerlik, afin, ve projektif yöntemleriyle iki farklı sisteme ait arazi koordinatları arasında datum dönüşümü örneklerine yer verilmiştir.

Her yöntem için yapılacak hesaplamalar MS Excel ortamında programlanmıştır. Hesaplamalarda her iki sistemdeki koordinatları bilinen beş dayanak noktası ile parametreler hesaplanarak dönüşümlere uygulanmıştır. İki farklı uygulama ile yöntemlerin verdiği sonuçlar ve dayanak noktalarının mevcut koordinatlar arasındaki konumları dikkate alınarak sonuçlar incelenmiştir.

Dönüşümde kullanılan uygulama noktaların arazi üzerindeki konumları ve uygulamalarda dayanak noktası olarak belirlenen noktalar Şekil 1,2 ve 3. ile gösterilmiştir. Uygulama noktalarının 1 . ve 2 . sisteme ait bilinen koordinatları, 'A' ve ' $L$ ' matrisleri makale sonunda yer alan 'EK-1' ile gösterilmiştir.

\section{BULGULAR}

Dönüşüm sonucunda her yöntem için bulunan parametreler ve ortalama hatalar Tablo 1. ile gösterilmiştir.

Elde edilen verilere göre her iki uygulama için, dönüşüm yöntemleri arasında en düşük ortalama hata miktarı projektif dönüşüm yönteminde gözlemlenmektedir. Ayrıca mevcut koordinat ağının dışındaki noktaları dayanak noktası olarak kullanan uygulama-1, koordinat ağının içerisinde kalan noktaları dayanak noktası olarak kullanan uygulama2'ye göre daha düşük ortalama hatalar ve düzeltme değerleri vermiştir. Dönüşümlere ait veriler Tablo 1, 2, 3 ve Tablo 4. üzerinde yer almaktadır.
Tablo 1. Dönüşüm Parametreleri ve Ortalama Hatalar

\begin{tabular}{|c|c|c|c|c|c|}
\hline Uyg.1 & $\begin{array}{l}\text { Benzerlik } \\
\text { Dönüşümü }\end{array}$ & & Afin Dönüşümü & & $\begin{array}{l}\text { Projektif } \\
\text { Dönüşümm }\end{array}$ \\
\hline a & 0,9999994172 & $\mathbf{a}$ & 0,9999997383 & a1 & 0,9999997718 \\
\hline b & $-0,0000050449$ & b & 0,0000051735 & b1 & 0,0000051927 \\
\hline c & 181,3745117188 & c & 179,9687500000 & c1 & 0,0004403814 \\
\hline \multirow[t]{5}{*}{ d } & 50,3291015625 & d & $-0,0000052924$ & a2 & $-0,0000052727$ \\
\hline & & e & 0,9999991575 & b2 & 0,9999991783 \\
\hline & & f & 51,5124511719 & c2 & $-0,0003950093$ \\
\hline & & & & a3 & $3,34524 \mathrm{E}-11$ \\
\hline & & & & b3 & $-4,66529 \mathrm{E}-12$ \\
\hline $\mathbf{m}_{0}=$ & $\pm 0.13 \mathrm{~cm}$ & & $\pm 0.34 \mathrm{~cm}$ & & $\pm 0.03 \mathrm{~cm}$ \\
\hline Uyg.2 & $\begin{array}{c}\text { Benzerlik } \\
\text { Dönüşümü̈ }\end{array}$ & & Afin Dönüşümü & & $\begin{array}{l}\text { Projektif } \\
\text { Dönüşümm }\end{array}$ \\
\hline $\mathbf{a}$ & 0,9999998650 & $\mathbf{a}$ & 0,9999999655 & a1 & 0,9999999328 \\
\hline b & $-0,0000054445$ & b & 0,0000056996 & b1 & 0,0000062115 \\
\hline c & 179,2799604178 & c & 178,7031250000 & c1 & 0,0008711331 \\
\hline \multirow[t]{5}{*}{ d } & 51,7163085938 & d & $-0,0000052875$ & a2 & $-0,0000050497$ \\
\hline & & e & 0,9999995123 & b2 & 0,9999992593 \\
\hline & & $\mathbf{f}$ & 51,2773437500 & c2 & 0,0002356464 \\
\hline & & & & a3 & $2,02944 \mathrm{E}-10$ \\
\hline & & & & b3 & $1,01134 \mathrm{E}-10$ \\
\hline $\mathbf{m}_{0}=$ & $\pm 0.30 \mathrm{~cm}$ & & $\pm 0.33 \mathrm{~cm}$ & & $\pm 0.03 \mathrm{~cm}$ \\
\hline
\end{tabular}

Tablo 2. Dönüşüm Sonrası Dayanak Noktalarının Düzeltme Miktarları

\begin{tabular}{|c|c|c|c|c|c|c|}
\hline Uyg.1 & \multicolumn{2}{|c|}{$\begin{array}{c}\text { Benzerlik } \\
\text { Dönüşümü }\end{array}$} & \multicolumn{2}{|c|}{ Afin Dönüşümü } & \multicolumn{2}{|c|}{$\begin{array}{l}\text { Projektif } \\
\text { Dönüşüm }\end{array}$} \\
\hline N.N. & $V_{X}(\mathbf{c m})$ & $V_{Y}(\mathbf{c m})$ & $\mathbf{V}_{\mathbf{X}}(\mathbf{c m})$ & $V_{Y}(\mathbf{c m})$ & $\mathbf{V}_{\mathbf{X}}(\mathbf{c m})$ & $\mathbf{V}_{\mathbf{Y}}(\mathbf{c m})$ \\
\hline N3230161 & $-0,138$ & 0,048 & 0,354 & 0,047 & 0,009 & 0,013 \\
\hline N3220003 & $-0,047$ & 0,041 & 0,284 & 0,040 & $-0,010$ & 0,013 \\
\hline N3230015 & $-0,183$ & 0,107 & 0,245 & 0,071 & $-0,006$ & $-0,022$ \\
\hline N3230019 & 0,099 & $-0,040$ & 0,351 & 0,060 & 0,014 & 0,001 \\
\hline N3230028 & $-0,044$ & $-0,149$ & 0,264 & 0,055 & $-0,008$ & $-0,004$ \\
\hline Uyg.2 & \multicolumn{2}{|c|}{$\begin{array}{c}\text { Benzerlik } \\
\text { Dönüșümü }\end{array}$} & \multicolumn{2}{|c|}{ Afin Dönüşümü } & \multicolumn{2}{|c|}{$\begin{array}{l}\text { Projektif } \\
\text { Dönüşüm }\end{array}$} \\
\hline N.N. & $V_{x}(\mathbf{c m})$ & $\mathbf{V}_{\mathbf{Y}}(\mathbf{c m})$ & $\mathbf{V}_{\mathbf{X}}(\mathbf{c m})$ & $\mathbf{V}_{\mathbf{Y}}(\mathbf{c m})$ & $V_{X}(\mathbf{c m})$ & $\mathbf{V}_{\mathbf{Y}}(\mathbf{c m})$ \\
\hline N3210001 & 0,426 & $-0,005$ & $-0,243$ & 0,007 & 0,009 & 0,004 \\
\hline N3230015 & 0,307 & 0,063 & $-0,248$ & 0,014 & $-0,013$ & 0,003 \\
\hline N3230016 & 0,261 & 0,007 & $-0,324$ & $-0,040$ & 0,030 & $-0,002$ \\
\hline N3230018 & 0,243 & $-0,002$ & $-0,382$ & $-0,041$ & $-0,030$ & $-0,008$ \\
\hline N3230019 & 0,344 & 0,131 & $-0,267$ & $-0,002$ & 0,004 & 0,002 \\
\hline
\end{tabular}


Tablo 3. Dönüşüm Sonrası Test Noktalarının Düzeltme Miktarları

\begin{tabular}{|c|c|c|c|c|c|c|}
\hline Uyg.-1 & \multicolumn{2}{|c|}{$\begin{array}{c}\text { Benzerlik } \\
\text { Dönüussümü }\end{array}$} & \multicolumn{2}{|c|}{ Afin Dönüşümü } & \multicolumn{2}{|c|}{$\begin{array}{l}\text { Projektif } \\
\text { Dönüşümm }\end{array}$} \\
\hline N.N.. & $\mathbf{V}_{\mathbf{x}}(\mathbf{c m})$ & $\mathbf{V}_{\mathbf{Y}}(\mathbf{c m})$ & $\mathbf{V}_{\mathbf{X}}(\mathbf{c m})$ & $\mathrm{V}_{\mathbf{Y}}(\mathbf{c m})$ & $\mathbf{V}_{\mathbf{x}}(\mathbf{c m})$ & $\begin{array}{r}\mathbf{V}_{\mathbf{Y}} \\
(\mathbf{c m})\end{array}$ \\
\hline N3210001 & $-0,206$ & $-0,053$ & $-0,489$ & $-0,178$ & $-0,211$ & $-0,073$ \\
\hline N3230016 & 0,145 & $-0,027$ & $-0,232$ & $-0,04$ & 0,021 & 0,048 \\
\hline N3230018 & 0,055 & 0,004 & $-0,259$ & $-0,073$ & 0,013 & 0,018 \\
\hline Uyg.-2 & \multicolumn{2}{|c|}{$\begin{array}{c}\text { Benzerlik } \\
\text { Dönüşümü }\end{array}$} & \multicolumn{2}{|c|}{ Afin Dönüşümü } & \multicolumn{2}{|c|}{$\begin{array}{l}\text { Projektif } \\
\text { Dönüşümm }\end{array}$} \\
\hline N.N. & $V_{X}(\mathbf{c m})$ & $\mathbf{V}_{\mathbf{Y}}(\mathrm{cm})$ & $\mathrm{V}_{\mathrm{X}}(\mathbf{c m})$ & $\mathbf{V}_{\mathbf{Y}}(\mathbf{c m})$ & $V_{X}(\mathbf{c m})$ & $\begin{array}{l}\mathbf{V}_{\mathbf{Y}} \\
(\mathbf{c m})\end{array}$ \\
\hline N3230161 & $-0,326$ & 0,395 & 0,323 & 0,194 & 0,799 & $-0,495$ \\
\hline N3220003 & $-0,363$ & $-0,27$ & 0,169 & $-0,07$ & $-0,409$ & 0,044 \\
\hline N3230028 & $-0,077$ & 0,605 & 0,714 & 0,329 & 0,681 & 0,273 \\
\hline
\end{tabular}

Tablo 4. Dayanak ve Test Noktalarının Her Yöntem İçin Maksimum-Minimum Düzeltme Miktarlar1

\begin{tabular}{|c|c|c|c|c|c|c|}
\hline \multicolumn{7}{|c|}{$\begin{array}{c}\text { Dayanak Noktalarının Maksimum ve Minimum Düzeltme } \\
\text { Miktarları }\end{array}$} \\
\hline Dönüşüm & \multicolumn{2}{|c|}{$\begin{array}{c}\text { Benzerlik } \\
\text { Dönüșümü }\end{array}$} & \multicolumn{2}{|c|}{\begin{tabular}{c|} 
Afin \\
Dönüșümü
\end{tabular}} & \multicolumn{2}{|c|}{$\begin{array}{l}\text { Projektif } \\
\text { Dönüşüm }\end{array}$} \\
\hline $\begin{array}{c}\text { Maksimum } \\
(\mathbf{V})\end{array}$ & $\begin{array}{l}\mathbf{V}_{\mathbf{X}} \\
(\mathrm{cm})\end{array}$ & $\begin{array}{c}\mathbf{V}_{\mathbf{Y}} \\
(\mathbf{c m})\end{array}$ & $\begin{array}{c}\mathbf{V}_{\mathbf{X}} \\
(\mathrm{cm})\end{array}$ & $\begin{array}{c}\mathbf{V}_{\mathbf{Y}} \\
(\mathbf{c m})\end{array}$ & $\begin{array}{l}\mathbf{V}_{\mathbf{X}} \\
(\mathbf{c m})\end{array}$ & $\begin{array}{c}\mathbf{V}_{\mathbf{Y}} \\
(\mathrm{cm})\end{array}$ \\
\hline Uyg. 1 & $-0,183$ & $-0,149$ & 0,354 & 0,071 & 0,014 & $-0,022$ \\
\hline Uyg.2 & 0,426 & 0,131 & $-0,382$ & $-0,041$ & 0,03 & $-0,008$ \\
\hline $\begin{array}{l}\text { Minimum } \\
(\mathbf{V})\end{array}$ & $\begin{array}{c}\mathbf{V}_{\mathbf{X}} \\
(\mathbf{c m})\end{array}$ & $\begin{array}{c}\mathbf{V}_{\mathbf{Y}} \\
(\mathbf{c m})\end{array}$ & $\begin{array}{c}\mathbf{V}_{\mathbf{x}} \\
(\mathrm{cm})\end{array}$ & $\begin{array}{c}\mathbf{V}_{\mathbf{Y}} \\
(\mathbf{c m})\end{array}$ & $\begin{array}{c}\mathbf{V}_{\mathbf{X}} \\
(\mathbf{c m})\end{array}$ & $\begin{array}{c}\mathbf{V}_{\mathbf{Y}} \\
(\mathbf{c m})\end{array}$ \\
\hline Uyg.1 & $-0,044$ & $-0,04$ & 0,245 & 0,04 & $-0,006$ & 0,001 \\
\hline Uyg.2 & 0,243 & $-0,002$ & $-0,243$ & $-0,002$ & 0,004 & 0,002 \\
\hline \multicolumn{7}{|c|}{ Test Noktalarının Maksimum ve Minimum Düzeltme Miktarları } \\
\hline Dönüşüm & \multicolumn{2}{|c|}{$\begin{array}{c}\text { Benzerlik } \\
\text { Dönüșümü }\end{array}$} & \multicolumn{2}{|c|}{$\begin{array}{c}\text { Afin } \\
\text { Dönüșümü }\end{array}$} & \multicolumn{2}{|c|}{$\begin{array}{l}\text { Projektif } \\
\text { Dönüșüm }\end{array}$} \\
\hline $\begin{array}{c}\text { Maksimum } \\
(\mathbf{V})\end{array}$ & $\begin{array}{l}\mathbf{V}_{\mathbf{X}} \\
(\mathbf{c m})\end{array}$ & $\begin{array}{c}\mathbf{V}_{\mathbf{Y}} \\
(\mathrm{cm})\end{array}$ & $\begin{array}{c}\mathbf{V}_{\mathbf{X}} \\
(\mathrm{cm})\end{array}$ & $\begin{array}{c}V_{Y} \\
(\mathbf{c m})\end{array}$ & $\begin{array}{c}V_{X} \\
(\mathbf{c m})\end{array}$ & $\begin{array}{c}\mathrm{V}_{\mathbf{Y}} \\
(\mathrm{cm})\end{array}$ \\
\hline Uyg. 1 & $-0,206$ & $-0,053$ & $-0,489$ & $-0,178$ & $-0,211$ & $-0,073$ \\
\hline Uyg.2 & $-0,363$ & 0,605 & 0,714 & 0,329 & 0,799 & $-0,495$ \\
\hline $\begin{array}{c}\text { Minimum } \\
(\mathbf{V})\end{array}$ & $\begin{array}{c}\mathrm{V}_{\mathbf{X}} \\
(\mathrm{cm})\end{array}$ & $\begin{array}{c}\mathbf{V}_{\mathbf{Y}} \\
(\mathrm{cm})\end{array}$ & $\begin{array}{c}\mathrm{V}_{\mathrm{X}} \\
(\mathrm{cm})\end{array}$ & $\begin{array}{c}\mathbf{V}_{\mathbf{Y}} \\
(\mathrm{cm})\end{array}$ & $\begin{array}{c}V_{X} \\
(\mathrm{~cm})\end{array}$ & $\begin{array}{c}\mathbf{V}_{\mathbf{Y}} \\
(\mathrm{cm})\end{array}$ \\
\hline Uyg.1 & 0,055 & 0,004 & $-0,232$ & $-0,04$ & 0,013 & 0,018 \\
\hline Uyg.2 & $-0,077$ & $-0,27$ & 0,169 & $-0,07$ & $-0,409$ & 0,044 \\
\hline
\end{tabular}

Dayanak noktaları için düzeltme değerleri arasında yukarı değer yönünde en yüksek düzeltme miktarı N3210001 numaralı noktada $0,426 \mathrm{~cm}$ ve sağa değer yönünde N3230019 numaral1 noktada $0,131 \mathrm{~cm}$ olarak belirlenmiştir. $\mathrm{Bu}$ düzeltme miktarları uygulama-2 sonrasında elde edilen benzerlik dönüşümü değerleridir. Dayanak noktaları için en düşük düzeltme miktarları ise projektif dönüşüm yöntemi ile elde edilmiş olup, yukarı yönde N3230019 numaralı noktada uygulama2 için $0,004 \mathrm{~cm}$ ve sağa değer yönünde N3230019 numaralı noktada uygulama-1 için $0,001 \mathrm{~cm}$ olarak belirlenmiştir.

Test noktaları için yukarı değer yönünde en yüksek düzeltme değeri uygulama-2 için projektif dönüşüm sonrasında N3230161 numaralı noktada $0,799 \mathrm{~cm}$ ve sağa değer yönünde N3230028 numaralı noktada uygulama-2 için benzerlik dönüşümü sonrasında $0,605 \mathrm{~cm}$ olarak belirlenmiştir. Test noktaları için en düşük düzeltme miktarları ise yukarı değer yönünde N3230018 numaralı noktada uygulama-1 için projektif dönüşüm sonrasında $0,013 \mathrm{~cm}$ ve sağa değer yönünde N3230018 numaralı noktada uygulama-1 için benzerlik dönüşümü sonrasında $0,004 \mathrm{~cm}$ olarak belirlenmiştir.

Hem dayanak hem de test noktaları için en yüksek ve en düşük düzeltme değerleri arasında afin dönüşüm yöntemine ait sonuç verisi yoktur. Afin dönüşüm yöntemi ile her iki uygulama için yakın değerler ve ortalama hatalar elde edilmiştir. Dayanak noktalarının koordinat ağındaki konumlarının durumu afin dönüşüm yöntemi için önemli farklılıklar göstermemektedir. Hesaplamalar sonucunda en yüksek ortalama hata uygulama-1 ve afin dönüşüm yöntemi ile $\pm 0.34 \mathrm{~cm}$ olarak ortaya çıkmıştır. En düşük ortalama hata ise uygulama-1 ve projektif dönüşüm yöntemi ile $\pm 0.03 \mathrm{~cm}$ olarak hesaplanmıştır. Benzerlik dönüşüm yöntemi ile ise uygulamalarda afin dönüşüm yöntemine göre daha düşük ortalama hatalar hesaplanmıştır ancak hesaplamalar sonucunda meydana gelen düzeltme miktarları arasinda önemli farkliliklar gözlemlenmektedir. Her yöntem için uygulama-1, uygulama-2'ye göre daha uygun sonuçlar vermiştir.

\section{SONUC VE ÖNERÍLER}

Uygulamalardan elde edilen bulgulara göre projektif dönüşüm yöntemi en düşük ortalama hata ve düzeltme miktarlarına sahip olan yöntem olarak belirlenmiştir. Afin dönüşüm yöntemi sonucunda ise hesaplanan düzeltme miktarları, her yöntem için ortaya çıkan maksimum ve minimum düzeltme miktarlarının arasında kalmaktadır. Hem afin hem de projektif dönüşüm yöntemi için 
uygulama-1 ve uygulama-2 sonucunda ortaya çıkan değerler önemli farkl1liklar göstermemektedir. Benzerlik dönüşümü için ise uygulamalar sonucunda önemli farkl1liklar görülmektedir. $\mathrm{Bu}$ nedenle arazi koordinatlarının diğer bir koordinat sistemine dönüşümünde bu çalışmada yapılan uygulamaların bulgularına göre projektif dönüşüm yöntemi ile daha uygun sonuçlar ortaya çıkmıştır. Projektif dönüşüm yöntemi için her iki sistemde koordinatları bilinen en az dört ortak nokta bilinmesi gerekirken afin dönüşüm yöntemi için en az üç bilinen ortak noktanın gerekmesi ve afin dönüşüm yönteminin projektif dönüşüm yöntemine göre uygulamalarda hesap kolaylığı sağlaması nedeniyle afin dönüşüm yöntemi de yapılan uygulamalar için uygun dönüşüm yöntemi olarak seçilebilir. Uygulama-1 ve uygulama-2 arasında yapılan karşılaştırmalara göre ise uygulama-1 de olduğu gibi dayanak noktalarının, koordinat ağını çevreleyen noktalardan seçilmesi daha uygun sonuçlar vermiştir. Koordinat dönüşümleri uygulanırken, dayanak noktalarının konumları ve uygulanacak yöntem hesaplamaların sonuçlarını doğrudan etkilemektedir. Uygulamaların bulgularına göre arazi koordinatlarının dönüşümü için en uygun yöntem projektif dönüşüm yöntemi olarak belirlenmiştir.

\section{KAYNAKÇA}

Aksoy, A. (1999). Jeodezide Değişimler. Harita ve Kadastro Mühendisliği Dergisi, 86, 40-60.

Akyılmaz, O., Acar, M., \& Özlüdemir, M. T. (2007). Koordinat Dönüşümünde En Küçük Kareler Ve Toplam En Küçük Kareler Yöntemleri. HKM Jeodezi, Jeoinformasyon ve Arazi Yönetimi Dergisi, 97, 15-22.

Başçiftçi, F., \& İnal, C. (2008). Jeodezide Kullanılan Bazı Koordinat Dönüşümlerinin Programlanması. J. Fac. Eng. Arch. Selcuk Univ, 23(1), 27-40.

Deniz, R., Çelik, R., N., Kutoğlu, H., Özlüdemir, M., T., Demir, C., \& Kınık, İ. (2012). Büyük Ölçekli Harita ve Harita Bilgileri Üretim Yönetmeliği: TMMOB Harita ve Kadastro Mühendisleri Odas1.

A DOD Glossary of Mapping, Charting and Geodetic Terms. (1967). Washington: U. S. Army Topographic Command.
Even-Tzur, G. (2000). Datum Definition for GPS Networks. Survey Review, 35(277), 475486.

Featherstone, W., \& Vanicek, P. (1999). The Role of Coordinate Systems, Coordinates and Heights in Horizontal Datum Transformations. Australian surveyor, 44(2), 143-150.

Geodetic Glossary. (1986). Rockville, MD: National Geodetic Survey.

Ghilani, C. D. (2011). Adjustment Computations: Spatial Data Analysis: John Wiley \& Sons.

Hofmann-Wellenhof, B., Lichtenegger, H., \& Wasle, E. (2007). GNSS - Global Navigation Satellite Systems (1 ed.): Springer-Verlag Wien.

Kahveci, M., \& Y1ld1z, F. (2012). GPS/GNSS: Uydularla Konum Belirleme Sistemleri: Teori ve Uygulama (5 ed.): Nobel.

Kraus, K. (2007). Fotogrametri Cilt 1 (O. Altan, S. Külür, G. Toz, H. Demirel, Z. Duran, \& M. Çelikoyan, Trans.). İstanbul Teknik Üniversitesi: Nobel Yayın Dağıtım.

Kwon, J. H., Bae, T.-S., Choi, Y.-S., Lee, D.-C., \& Lee, Y.-W. (2005). Geodetic Datum Transformation to The Global Geocentric Datum for Seas and Islands Around Korea. Geosciences Journal, 9(4), 353-361.

Mitsakaki, C., Agatza-Balodimou, A., \& Papazissi, K. (2006). Geodetic Reference Frames Transformations. Survey Review, 38(301), 608-618.

Ogaja, C. A. (2011). Applied GPS for Engineers and Project Managers.

Şişman, Y., \& Dilaver, A. (2005). Datum Dönüşümünde Kalite Kontrol. Paper presented at the 10. Türkiye Harita Bilimsel ve Teknik Kurultayı, Ankara.

Uzun, Y. (2003). Üç Boyutlu Astrojeodezik Dik Koordinat Sistemlerinde Dönüşüm Modelleri ve Uyuşumsuz Ölçü Gruplarının Belirlenmesi Yöntemlerinin Karşılaştırılması. (Doktora Tezi), Karadeniz Teknik Üniversitesi, Trabzon.

Üstün, A. (1996). Datum Dönüşümleri. (Yüksek Lisans Tezi), Yıldız Teknik Üniversitesi, İstanbul.

Vaníček, P., \& Steeves, R. R. (1996). Transformation of Coordinates Between Two Horizontal Geodetic Datums. Journal of Geodesy, 70(11), 740-745. 


\section{EK-1}

Uygulama Noktalarının 1. ve 2. Sisteme Ait Bilinen Koordinatları;

\begin{tabular}{|l|l|c|c|c|c|}
\hline \multirow{2}{*}{ Nokta No } & \multirow{2}{*}{ Uygulama-1 } & \multicolumn{2}{|c|}{ 1. Sistem Koordinatlar1 } & \multicolumn{2}{c|}{ 2. Sistem Koordinatlar1 } \\
\cline { 3 - 6 } & & $\mathbf{x}(\mathbf{m})$ & $\mathbf{y}(\mathbf{m})$ & $\mathbf{X}(\mathbf{m})$ & $\mathbf{Y}(\mathbf{m})$ \\
\hline N3230161 & Dayanak Noktas1 & 4153869,344 & 598620,722 & 4154051,319 & 598649,746 \\
\hline N3220003 & Dayanak Noktas1 & 4145749,901 & 606385,748 & 4145931,919 & 606414,808 \\
\hline N3230015 & Dayanak Noktas1 & 4149774,186 & 603885,358 & 4149956,190 & 603914,399 \\
\hline N3230019 & Dayanak Noktas1 & 4144342,828 & 603836,344 & 4144524,833 & 603865,414 \\
\hline N3230028 & Dayanak Noktas1 & 4149760,923 & 594664,170 & 4149942,880 & 594693,219 \\
\hline N3210001 & Test Noktasl & 4146561,245 & 600716,849 & 4146743,231 & 600745,908 \\
\hline N3230016 & Test Noktasl & 4148459,658 & 603253,359 & 4148641,660 & 603282,408 \\
\hline N3230018 & Test Noktasl & 4146865,503 & 602317,171 & 4147047,500 & 602346,229 \\
\hline
\end{tabular}

\begin{tabular}{|l|l|c|c|c|c|}
\hline \multirow{2}{*}{ Nokta No } & \multirow{2}{*}{ Uygulama-2 } & \multicolumn{2}{|c|}{ 1. Sistem Koordinatlar1 } & \multicolumn{2}{c|}{ 2. Sistem Koordinatlar1 } \\
\cline { 3 - 6 } & & $\mathbf{x}(\mathbf{m})$ & $\mathbf{y}(\mathbf{m})$ & $\mathbf{X}(\mathbf{m})$ & Y $(\mathbf{m})$ \\
\hline N3210001 & Dayanak Noktas1 & 4146561,245 & 600716,849 & 4146743,231 & 600745,908 \\
\hline N3230015 & Dayanak Noktas1 & 4149774,186 & 603885,358 & 4149956,190 & 603914,399 \\
\hline N3230016 & Dayanak Noktas1 & 4148459,658 & 603253,359 & 4148641,660 & 603282,408 \\
\hline N3230018 & Dayanak Noktas1 & 4146865,503 & 602317,171 & 4147047,500 & 602346,229 \\
\hline N3230019 & Dayanak Noktas1 & 4144342,828 & 603836,344 & 4144524,833 & 603865,414 \\
\hline N3230161 & Test Noktası & 4153869,344 & 598620,722 & 4154051,319 & 598649,746 \\
\hline N3220003 & Test Noktasl & 4145749,901 & 606385,748 & 4145931,919 & 606414,808 \\
\hline N3230028 & Test Noktasl & 4149760,923 & 594664,170 & 4149942,880 & 594693,219 \\
\hline
\end{tabular}

\section{Uygulamalarda Kullanılan 'A' ve ' $L$ ' Matrisleri;}

Benzerlik Dönüşümü (Uygulama-1) ;

$A=\quad \begin{array}{cc}4153869,344 & -598620,722 \\ 598620,722 & 4153869,344 \\ 4145749,901 & -606385,748 \\ 606385,748 & 4145749,901 \\ 4149774,186 & -603885,358 \\ 603885,358 & 4149774,186 \\ 4144342,828 & -603836,344 \\ 603836,344 & 4144342,828 \\ 4149760,923 & -594664,170 \\ 594664,170 & 4149760,923\end{array}$

Afin Dönüşümü (Uygulama-1) ;

\begin{tabular}{ll|l}
1 & 0 \\
0 & 1 \\
1 & 0 \\
0 & 1 \\
1 & 0 \\
0 & 1 & \\
1 & 0 \\
0 & 1 & \\
1 & 0 & \\
0 & 1 &
\end{tabular}

$(10 \times 4)$

$$
\begin{array}{|c|}
4154051,319 \\
598649,746 \\
4145931,919 \\
606414,808 \\
4149956,19 \\
603914,399 \\
4144524,833 \\
603865,414 \\
4149942,88 \\
594693,219
\end{array}
$$

$(10 x 1)$

$$
\begin{array}{|cccccc|}
4153869,344 & 598620,722 & 1 & 0 & 0 & 0 \\
0 & 0 & 0 & 4153869,344 & 598620,722 & 1
\end{array} \mid
$$




$\mathrm{A}=\left|\begin{array}{cccccc}4145749,901 & 606385,748 & 1 & 0 & 0 & 0 \\ 0 & 0 & 0 & 4145749,901 & 606385,748 & 1 \\ 4149774,186 & 603885,358 & 1 & 0 & 0 & 0 \\ 0 & 0 & 0 & 4149774,186 & 603885,358 & 1 \\ 4144342,828 & 603836,344 & 1 & 0 & 0 & 0 \\ 0 & 0 & 0 & 4144342,828 & 603836,344 & 1 \\ 4149760,923 & 594664,170 & 1 & 0 & 0 & 0 \\ 0 & 0 & 0 & 4149760,923 & 594664,170 & 1\end{array}\right|$

$(10 \times 6)$

$L=\left|\begin{array}{c}4154051,319 \\ 598649,746 \\ 4145931,919 \\ 606414,808 \\ 4149956,19 \\ 603914,399 \\ 4144524,833 \\ 603865,414 \\ 4149942,88 \\ 594693,219\end{array}\right|$

$(10 \times 1)$

Projektif Dönüşüm (Uygulama-1) ;

$\mathrm{A}=\left|\begin{array}{cccccc}5169,90748 & -2857,74632 & 1 & 0 & 0 & 0 \\ 0 & 0 & 0 & 5169,90748 & -2857,74632 & 1 \\ -2949,53552 & 4907,27918 & 1 & 0 & 0 & 0 \\ 0 & 0 & 0 & -2949,53552 & 4907,27918 & 1 \\ 1074,74908 & 2406,88968 & 1 & 0 & 0 & 0 \\ 0 & 0 & 0 & 1074,74908 & 2406,88968 & 1 \\ -4356,60802 & 2357,87578 & 1 & 0 & 0 & 0 \\ 0 & 0 & 0 & -4356,60802 & 2357,87578 & 1 \\ 1061,48698 & -6814,29832 & 1 & 0 & 0 & 0 \\ 0 & 0 & 0 & 1061,48698 & -6814,29832 & 1\end{array}\right|_{(10 \times 6)}$




$$
\mathrm{L}=\left|\begin{array}{c}
-2949,5092 \\
4907,2908 \\
1074,7618 \\
2406,8818 \\
-4356,5952 \\
2357,8968 \\
1061,4518 \\
-6814,2982
\end{array}\right|
$$

$(10 \times 1)$

Helmert Dönüşümü (Uygulama-2) ;

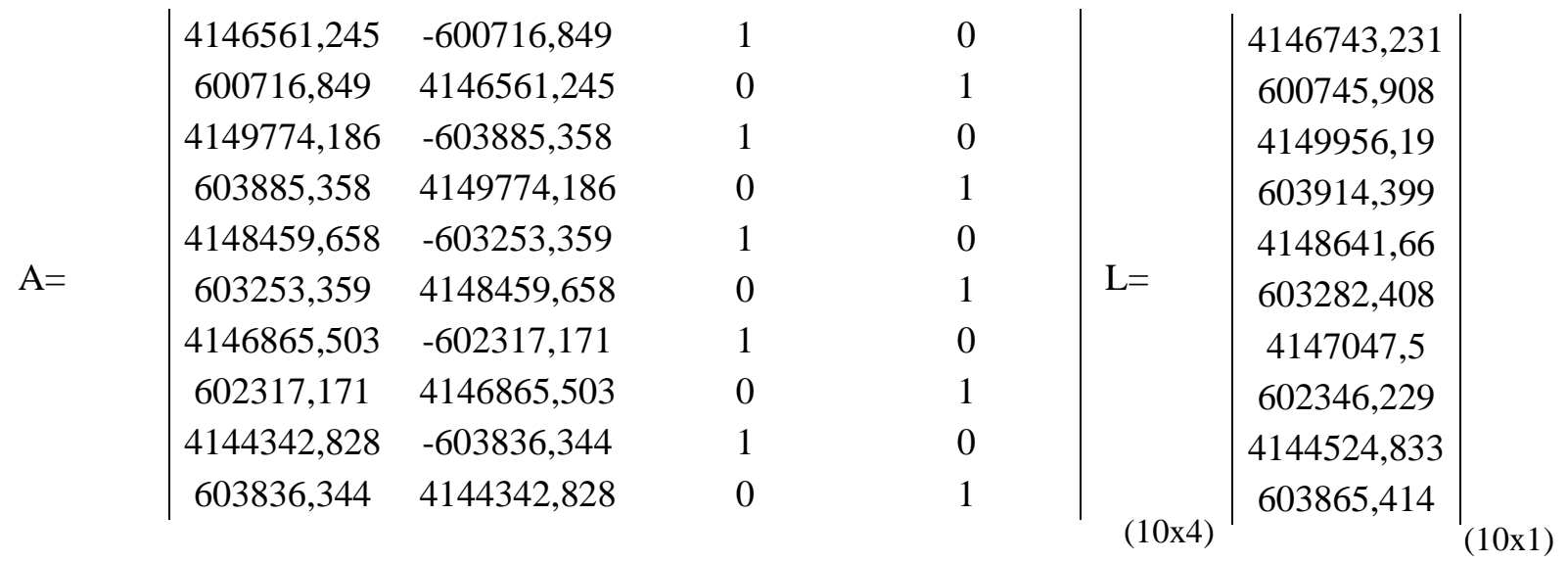

Afin Dönüşümü (Uygulama-2) ;

$\mathrm{A}=\left|\begin{array}{cccccc}4146561,245 & 600716,849 & 1 & 0 & 0 & 0 \\ 0 & 0 & 0 & 4146561,245 & 600716,849 & 1 \\ 4149774,186 & 603885,358 & 1 & 0 & 0 & 0 \\ 0 & 0 & 0 & 4149774,186 & 603885,358 & 1 \\ 4148459,658 & 603253,359 & 1 & 0 & 0 & 0 \\ 0 & 0 & 0 & 4148459,658 & 603253,359 & 1 \\ 4146865,503 & 602317,171 & 1 & 0 & 0 & 0 \\ 0 & 0 & 0 & 4146865,503 & 602317,171 & 1 \\ 4144342,828 & 603836,344 & 1 & 0 & 0 & 0 \\ 0 & 0 & 0 & 4144342,828 & 603836,344 & 1\end{array}\right|_{(10 \times 6)}$




$$
\mathrm{L}=\left|\begin{array}{c}
4146743,231 \\
600745,908 \\
4149956,19 \\
603914,399 \\
4148641,66 \\
603282,408 \\
4147047,5 \\
602346,229 \\
4144524,833 \\
603865,414
\end{array}\right|_{(10 x 1)}
$$

Projektif Dönüşüm (Uygulama-2) ;

$\mathrm{A}=\left|\begin{array}{cccccc}-639,43944 & -2084,9678 & 1 & 0 & 0 & 0 \\ 0 & 0 & 0 & -639,43944 & -2084,9678 & 1 \\ 2573,50156 & 1083,5418 & 1 & 0 & 0 & 0 \\ 0 & 0 & 0 & 2573,50156 & 1083,5418 & 1 \\ 1258,97436 & 451,543 & 1 & 0 & 0 & 0 \\ 0 & 0 & 0 & 1258,97436 & 451,543 & 1 \\ -335,18094 & -484,6449 & 1 & 0 & 0 & 0 \\ 0 & 0 & 0 & -335,18094 & -484,6449 & 1 \\ -2857,85554 & 1034,5279 & 1 & 0 & 0 & 0 \\ 0 & 0 & 0 & -2857,85554 & 1034,5279 & 1\end{array}\right|$ (10x6)

$$
\mathrm{L}=\mid \begin{gathered}
-639,4518 \\
-2084,9636 \\
2573,5072 \\
1083,5274 \\
1258,9772 \\
451,5364 \\
-335,1828 \\
-484,6426 \\
-2857,8498 \\
1034,5424
\end{gathered}
$$

(10x1) 